\title{
QUANTUM PLANES AND QUANTUM CYLINDERS FROM POISSON HOMOGENEOUS SPACES.
}

\author{
N. Ciccoli \\ Faculteit der Wiskunde en Informatica, $\dagger$ \\ Universiteit van Amsterdam. \\ The Netherlands.
}

\begin{abstract}
Quantum planes and a new quantum cylinder are obtained as quantization of Poisson homogeneous spaces of two different Poisson structures on classical euclidean group $\mathrm{E}(2)$.

Mathematics Subject Classification (1991). 17B, 81R50.
\end{abstract}

\section{Introduction.}

The concept of homogeneous space of a group is maybe one of the most widespread mathematical concept, lying, for example, at the very foundation of harmonic analysis and of symmetries of physical systems. From the very beginning of the theory of quantum groups, clarifying the concept of a quantum homogeneous space for a quantum group has been considered of the uttermost importance (see [11] for example), although up to now a complete theory is still lacking. The purpose of this work is to study homogeneous quantum spaces of euclidean quantum groups through the analysis of the "semiclassical" limit Poisson structure on the classical group. Our aim is to verify to what extent the results in [12] and [13] relating covariant Poisson structures on the sphere and the one parameter family of quantum spheres [11] are still valid in this case (although we will not deal with the analytical aspects as it is extensively done in those references). There are two

\footnotetext{
$\dagger$ on leave from Dipartimento di Matematica, Universitá di Bologna. Italy.
} 
different versions of the Euclidean quantum group, introduced in [15] and [3] respectively; in this second reference it is also shown how they can be both obtained through contraction procedure from $S U_{h}(2)$. The way in which they are related is explained in [1]. Quite a lot of work has been done on these groups, whose interest lies both in being an easy example of the nonsemisimple and noncompact case and in physical applications. For example in [16] the standard euclidean quantum group is treated at an analytical level, in [15] its relations with $q$-special functions are first studied and later in [2] and [9] (last of a series of paper and useful for further references) $q$-harmonic analysis is more thouroghly investigated. The roots of unity theory has been dealt with in [5] for the standard case, and has been claimed and proved to be trivial in the non standard case respectively in [1] and [4]. The quantum homogeneous spaces have been studied in the standard case in [2], through duality arguments involving the quantized universal enveloping algebra. The semiclassical limit of these results is here shown to fit in the framework of the classification of covariant Poisson structures. This is the content of section 2. Furthermore in the nonstandard case the semiclassical limit suggests quite naturally that homogeneous spaces should be of cylinder-type. The covariant Poisson structures on this cylinder are classified in section 3 and in section 4 an explicit quantum cylinder is given through generators and relations, verifying conditions explained in [6] for quantum homogeneous spaces.

\section{The standard Poisson algebraic $\mathcal{E}(2)$ and quantum planes.}

The classical 2-dimensional Euclidean group is usually written in complex notation as the space of matrices of the form:

$$
\left(\begin{array}{ll}
v & n \\
0 & 1
\end{array}\right)
$$

Its function (polynomial) algebra can thus be seen as the commutative algebra on generators $v, \bar{v}, n, \bar{n}$, with the additional relation $v \bar{v}=1$. The matrix form gives us immediatly also the Hopf algebra structure that we explicitly write down as follows:

$$
\begin{gathered}
\Delta v=v \otimes v \quad \Delta \bar{v}=\bar{v} \otimes \bar{v} \\
\Delta n=\bar{v} \otimes n+n \otimes 1 \quad \Delta \bar{n}=v \otimes \bar{n}+\bar{n} \otimes 1 \\
S(v)=\bar{v} \quad S(n)=-v n \quad S(\bar{n})=-\bar{v} \bar{n} \\
\varepsilon(v)=1 \quad \varepsilon(n)=\varepsilon(\bar{n})=0
\end{gathered}
$$


and the usual $*$-structure is given by:

$$
v^{*}=\bar{v} \quad n^{*}=\bar{n} \quad *^{2}=I d
$$

Let us define on this function algebra the following quadratic Poisson bracket (standard bracket in what follows):

$$
\begin{aligned}
\{v, n\}=v n & \{v, \bar{n}\}=v \bar{n} \\
\{n, \bar{n}\}=n \bar{n} & \{v, \bar{v}\}=0
\end{aligned}
$$

(2.1) REMARK. These formulas define a Poisson bracket on the algebra of polynomial functions on $\mathcal{E}(2)$. That's why we talk about an algebraic Poisson structure and not of a Lie-Poisson structure that should be given on the whole algebra of smooth functions. In the following we will some times drop the adjective algebraic.

(2.2) Proposition. The formulas above define a Poisson algebraic structure on $\mathcal{E}(2)$.

Proof. Let us write the Poisson bivector corresponding to the bracket as:

$$
w(v, n, \bar{n})=v n \partial_{v} \wedge \partial_{n}+v \bar{n} \partial_{v} \wedge \partial_{\bar{n}}+n \bar{n} \partial_{n} \wedge \partial_{\bar{n}}
$$

By direct computation this Poisson bivector verifies the multiplicativity property:

$$
w\left(g g^{\prime}\right)=\left(\left(L_{g}\right)_{g^{\prime}}^{\prime} \otimes\left(L_{g}\right)_{g^{\prime}}^{\prime}\right) w\left(g^{\prime}\right)+\left(\left(R_{g^{\prime}}\right)_{g}^{\prime} \otimes\left(R_{g^{\prime}}\right)_{g}^{\prime}\right)(w(g))
$$

where $L_{g}$ and $R_{g}$ stands respectively for left and right translations in $\mathcal{E}(2)$.

Differentiating the Poisson bivector at the origin gives a coalgebra structure on the Lie algebra $e(2)$. Choosing $J=\partial_{v}, X=\partial_{n}, Y=\partial_{\bar{n}}$ as generators of the Lie algebra (obviously considering them around the identity) we have the coalgebra structure:

$$
\begin{gathered}
\delta(J)=0 \quad \delta(X)=J \wedge X \\
\delta(Y)=J \wedge Y
\end{gathered}
$$

It is easy to show that such structure is non-coboundary.

The Poisson bivector above mentioned defines the associated homomorphism $B_{w}$ from the cotangent to the tangent bundle of $\mathcal{E}(2)$ that can be easily calculated to be:

$$
d v \mapsto-v\left(n \partial_{n}+\bar{n} \partial_{\bar{n}}\right)=X_{v}
$$




$$
\begin{gathered}
d n \mapsto n\left(-v \partial_{v}+\bar{n} \partial_{\bar{n}}\right)=X_{n} \\
d \bar{n} \mapsto \bar{n}\left(n \partial_{n}+v \partial_{v}\right)=X_{\bar{n}}
\end{gathered}
$$

Thus we have the differentiable distribution of tangent subspaces:

$$
(v, n, \bar{n}) \rightarrow\left(X_{v}, X_{n}, X_{\bar{n}}\right)
$$

whose integral manifolds are the symplectic leaves of the Poisson structure.

(2.3) Remark. Geometric description of symplectic leaves. Let us observe that for a generic point of the space $(v, n, \bar{n})$ we have :

$$
v n \bar{n} X_{v}+\bar{n} X_{n}+n X_{\bar{n}}=0
$$

and thus the tangent space is generically 2-dimensional.

All the points of the form $(v, 0,0)$ are 0 -dim symplectic leaves of this Poisson distribution. The set of all this point is nothing but $\mathbf{S}^{1}$ viewed as a subgroup of $\mathcal{E}(2)$, and thus, being an union of leaves, it is a Poisson algebraic subgroup. It is not difficult to see that this subgroup, together with all its finite subgroup are the only Poisson algebraic subgroup of $\mathcal{E}(2)$.

At this point it could be interesting to calculate the primitive spectrum of standard $\operatorname{Fun}_{q}(\mathcal{E}(2))$ to see if it is in a good relation with the symplectic foliation. The primitive spectrum of this algebra is not very difficult to explicit after we've noticed that it can be given the form of an iterated skew (twisted) polynomial ring (see [7], [8]).

(2.4) Corollary. The $\mathcal{E}(2)$-homogeneous space $\mathbf{R}^{2}$ has a natural Poisson structure induced by the algebraic Poisson structure on $\mathcal{E}(2)$.

The projection map $\pi: \mathcal{E}(2) \rightarrow \mathbf{R}^{2}$ is nothing but the map that sends $(v, n, \bar{n}) \rightarrow\left(\frac{1}{2}(n+\bar{n}),-i \frac{1}{2}(n-\bar{n})\right)$. Calling $z$ and $\bar{z}$ the coordinates on the plane it is then immediate that the Poisson structure on the plane is:

$$
\{z, \bar{z}\}=z \bar{z}
$$

By definition such a structure is $(\mathcal{E}(2), w)$-covariant. We recall that by covariance we mean that the action map $\phi: \mathcal{E}(2) \times \mathbf{R}^{2} \rightarrow \mathbf{R}^{2}$ is a Poisson map when the first space is given the product Poisson structure.

(2.5) Remark. The Poisson structure just defined on the plane has a symplectic foliation consisting in two families of $0-\operatorname{dim}$ leaves parametrized by points 
of two orthogonal lines and four $2-\operatorname{dim}$ leaves separated by those points. Observing that the usual quantum plane structure $F_{q}$, the algebra on two $q$-commuting generators, is a quantization of this Poisson bracket, it's no surprise that the primitive spectrum of this algebra is in bijective correspondence with the foliation (see [14] for the explicit expression of the primitive spectrum).

The next problem is that of classifying all $(\mathcal{E}(2), w)$-covariant Poisson structures on the plane. We will follow [17] where the covariance condition is rewritten at the infinitesimal level. We will denote with:

$$
\phi: \mathcal{E}(2) \times \mathbf{R}^{2} \rightarrow \mathbf{R}^{2}
$$

the action map, with $\phi_{x}=\phi(\cdot, x) \quad \forall x \in R^{2}$ and $\phi_{g}=\phi(g, \cdot) \quad \forall g \in \mathcal{E}(2)$. With these notations condition 2.2 of [17] states that covariant structures on the plane are in 1-1 correspondence with elements $\rho \in \bigwedge^{2} T_{0}\left(\mathbf{R}^{2}\right)$ such that

$$
\left(\phi_{0}\right)_{*, e} \delta(X)+X \cdot \rho=0 \quad \forall X \in \mathcal{G}_{0}
$$

where $\mathcal{G}_{0}$ is the tangent algebra of the rotation subgroup - the stabilizer of 0 in $\mathbf{R}^{2}$. In fact such a $\rho$ can be extended to a Poisson bivector field on $\mathbf{R}^{2}$ simply as:

$$
\rho(x)=\left(\phi_{0}\right)_{*, g}(w(g))+\left(\phi_{g}\right)_{*, 0}(\rho)
$$

where $g \in \mathcal{E}(2)$ is such that $g \cdot 0=x$. Now, as $\mathcal{G}_{0}$ is nothing but the algebra generated by $J$ and $\delta(J)=0$, the above condition rewrites as $J \cdot \rho=0$ (simply an invariance condition) which is always verified. We can then take $\rho=k \partial_{1} \wedge \partial_{2}$, where the derivatives are calculated in 0 . The corresponding bivector field is:

$$
(a, b) \mapsto k \partial_{1} \wedge \partial_{2}
$$

and the Poisson bracket on the plane is:

$$
\{z, \bar{z}\}=z \bar{z}+k \text {. }
$$

Note that when $\rho=0$ we obtain exactly the Poisson structure on the plane given by Corollary 2.4. We have then just proved the following

(2.6) Proposition. There is a one-parameter family of covariant Poisson structures on the plane, with respect to the standard Poisson structure on $\mathcal{E}(2)$, given by:

$$
\{z, \bar{z}\}=z \bar{z}+k \text {. }
$$


(2.7) Remark. In the case in which $k \neq 0$ the symplectic foliation changes drastically. The 0 -dimensional symplectic leaves are the points of the hyperbola $z \bar{z}=-k$, and they divide the plane in three 2-dimensional symplectic leaf. Following this geometric picture we will refer to this case as the case of hyperbolic covariant structures.

\section{The nonstandard Poisson structure and the cylinder.}

We still deal with the same function algebra but we want to consider another family of Poisson structure (to which we'll refer as non standard Poisson structures) given in [10] and whose quantization gives the so-called non standard euclidean quantum group.

$$
\begin{gathered}
\{v, n\}=\omega(1-v) \\
\{v, \bar{n}\}=-\omega\left(v^{2}-v\right) \\
\{n, \bar{n}\}=\omega(n-\bar{n})
\end{gathered}
$$

where $\omega$ is a nonzero complex number.

That this bracket gives a Poisson algebraic structure on $\mathcal{E}(2)$ (or better a family of isomorphic structures) is proven in [10].

The infinitesimal counterpart of the bracket is the coproduct on the Lie algebra $e(2)$ given by:

$$
\delta\left(P_{1}\right)=0 \quad \delta\left(P_{2}\right)=\omega P_{2} \wedge P_{1} \quad \delta(J)=\omega J \wedge P_{2} .
$$

This is a coboundary coproduct by taking:

$$
\delta(X)=a d_{X} r, \quad r=\omega J \wedge P_{2} .
$$

We will call $w^{\prime}$ the Poisson bivector and $B_{w^{\prime}}$ the associated homomorphism between tangent and cotangent spaces is:

$$
\begin{gathered}
d v \mapsto \omega(v-1) \partial_{n}+\omega\left(v^{2}-v\right) \partial_{\bar{n}}=\omega(v-1)\left(\partial_{n}+v \partial_{\bar{n}}\right)=X_{v} \\
d n \mapsto \omega(1-v) \partial_{v}+\omega(\bar{n}-n) \partial_{\bar{n}}=X_{n} \\
d \bar{n} \mapsto \omega\left(v-v^{2}\right) \partial_{v}+\omega(n-\bar{n}) \partial_{n}=X_{\bar{n}}
\end{gathered}
$$


from which we have the distribution of tangent subspaces that integrates to simplectic leaves.

(3.1) Remark. For every $(v, n, \bar{n})$ the relation:

$$
\left(v-v^{2}\right) X_{n}+(v-1) X_{\bar{n}}+(\bar{n}-n) X_{v}=0
$$

holds, showing that the distribution is at most two-dimensional at every point. If we restrict to point of $\mathbf{S}^{1}$ given by $n=\bar{n}=0$ as in the previous case, we see that the distribution is exactly two-dimensional in these points and thus every point of the form $(v, 0,0)$ is contained in a two-dimensional symplectic leaf. Thus $\mathbf{S}^{1}$ is not a Poisson subgroup of non standard Poisson $\mathcal{E}(2)$. If we restrict to points $v=1$ and $n=\bar{n}$ we see that the distribution vanish and thus all these points are $0-\operatorname{dim}$ symplectic leaves. Again we're dealing with a subgroup (that in the matrix form corresponds to upper triangular unimodular $2 \times 2$ real matrices) and it is isomorphic to $\mathbf{R}$. Thus $\mathbf{R}$ is a Poisson subgroup for the non standard Poisson structure on $\mathcal{E}(2)$. This implies that the corresponding homogeneous space, a cylinder, has a canonical covariant Poisson structure. One further remark could be that in this case also the discrete infinite groups are Poisson subgroup. However we have to notice that, as the Poisson structure is defined only at the algebraic level, such subgroups are not closed, thus can not be given by annhilating an element of the function algebra.

Again it may be interesting to confront the whole symplectic foliation with the primitive ideal structure of its quantization, to compute which one could use the fact that $\mathcal{E}(2)$ is an iterated differential polynomial ring (as noted in [4]).

(3.2) Proposition. The cylinder $\mathcal{C}$, viewed as a homogeneous space of the non standard Poisson Euclidean group, can be parametrized as having the function algebra generated by $v, \bar{v}$ and $\bar{v} \bar{n}-v n=m$ with the covariant Poisson structure given by:

$$
\{v, m\}=-\omega\left(v^{2}-1\right)
$$

Proof. It is enough to show that the two functions $v$ and $m$ parametrize the cylinder, the rest will follow immediatly. That the function $v$ is invariant with respect to the action of $\mathcal{A}$ is obvious, and an easy calculation shows also the invariance of $m$.

(3.3) Remark. From the explicit Poisson bracket of 3.3 one can obtain the corresponding symplectic foliation on the cylinder. The distribution of tangent 
subspaces is:

$$
\begin{gathered}
d v \mapsto \omega\left(v^{2}-1\right) \partial_{m}=X_{v} \\
d m \mapsto-\omega\left(v^{2}-1\right) \partial_{v}=X_{m}
\end{gathered}
$$

and thus all the points on the line $v=1$ are $0-d i m$ symplectic leaves and all the other points belong to a unique 2 - dim leaf.

(3.4) REMARK. Now we want to give a classification of covariant structures on the cylinder as in 2.6. Again multiplicative $\left(\mathcal{E}(2), w^{\prime}\right)$-Poisson structures are characterized by their value in one point, say $v=1, m=0$. In this point a bivector has the form $\varrho=k \partial_{v} \wedge \partial_{m}$ and, with notations as in 2.5 , has to fulfill the invariance condition:

$$
\left(\phi_{(1,0)}\right)_{*} \delta\left(P_{1}\right)+P_{1} \cdot \varrho=0
$$

that reduces to the condition $P_{1} \cdot \varrho=0$ due to triviality of $\delta\left(P_{1}\right)$. This condition is always verified. This implies that the space of covariant Poisson structures has the form of an affine 1-dimensional space given adjoining to the structure of Proposition 3.2 any invariant bivector field extending $\varrho$. Explicitely the possible Poisson structures are listed in the following:

(3.5) Proposition. There is a one parameter family of covariant Poisson structures on the cylinder, with respect to the non standard Poisson $\mathcal{E}(2)$, given by:

$$
\{v, m\}=-\omega\left(v^{2}-1\right)+k .
$$

(3.6) REMARK. As for the plane the case $k \neq 0$ shows a completely different symplectic foliation. Just remark that the bracket is trivial when:

$$
\omega v^{2}+\omega+k=0
$$

from which we obtain the solutions:

$$
v= \pm \sqrt{\left(1+\omega^{-1} k\right)}
$$

The condition that $v$ is on the unit circle can be expressed as $v \bar{v}=1$. Except from the singular situation in which $\omega^{-1} k \in i \mathbf{R}$, there is always a special value of $k \neq 0$ for which $1+\omega^{-1} k$ (and thus its square roots) belong to the unit circle. Thus for every complex $\omega$ which is not purely imaginary there are always two values of $v$ for which the Poisson bracket is degenerate. The corresponding symplectic foliation 
is then given by two lines of points and two $2-\operatorname{dim}$ symplectic leaves between them. For all the other values of $k$ the distribution is two dimensional in every point and so the Poisson bracket induces a symplectic form on the whole cylinder.

\section{Quantum homogeneous cylinder}

We want now to pass from the semiclassical situation of Poisson homogeneous spaces to quantum homogeneous spaces, as defined for example in [6]. The standard $\mathcal{E}_{\omega}(2)$ has been treated in [2], where connections with the theory of spherical functions are also explained, so that in what follows we will restrict to the nonstandard case where the quantization of the given Poisson structure can be obtained, as in [10], simply substituting the Poisson bracket with the commutator, leaving the coalgebra structure unchanged. Let us just remark the connections between [2] and Proposition 2.6. The natural covariant Poisson structure is exactly the semiclassical limit of the usual quantum plane, and is, in fact the only quantum homogeneous spaces obtained by "quotient" with respect to a proper quantum subgroup, incidentally the quantization of the unique Poisson subgroup of $\mathcal{E}(2)$. The quantum hyperboloid of [2] has as semiclassical limit the covariant Poisson structure of 2.6 with $k=-2$ (and the analysis of symplectic leaves give a clear meaning to the appearing of the world "hyperbolic" in its name). The fact that covariant Poisson structures constitutes a complete one-parameter family either suggests the possibility of other quantum planes of which the two given in [13] should be, in a sense, paradigmatic, or asks for an explanation of the failure of quantizing Poisson homogeneous spaces for values of $k$ different from 0 and -2 .

Let's move to the nonstandard case. Let us recall ([1]) that the nonstandard quantum group is the Hopf algebra with the same coproduct, counit and antipode as the standard quantum group and commutation relations obtained substituting the Poisson bracket with the Lie bracket in formulas at the beginning of chapter 3 .

The classical projection $\pi: G \rightarrow M$ from one Poisson-Lie group to its homogeneous space correspond to an injective map on the function algebra level $\hat{\pi}: \mathcal{F}(M) \rightarrow \mathcal{F}(G)$. The remark just stated above says that we can see this map as a map between the quantizations and requiring it to be an algebra map we find the quantized commutation relations for the Poisson homogeneous space. It is obvious how it works in the quantum plane case. This implies that we can give the following: 
(4.1) Definition. The quantum cylinder $\mathcal{C}_{\omega}$ is the algebra generated by the elements $v, \bar{v}$ and $m$ with the relations:

$$
v \bar{v}=\bar{v} v=1 \quad v m=m v-\omega\left(v^{2}-1\right) \quad \bar{v} m=m \bar{v}+\omega\left(\bar{v}-\bar{v}^{2}\right)
$$

and with the *-structure:

$$
v^{*}=\bar{v} \quad m^{*}=-m
$$

(4.2) Proposition. The quantum cylinder is a quantum homogeneous space of nonstandard $\mathcal{E}_{\omega}(2)$ in the sense of [6] i.e. it is a*-invariant right coideal . Furthermore the elements $\left\{v^{r} m^{s}: r \in \mathbf{Z}, s \in \mathbf{N}\right\}$ provide a basis as a vector space for this algebra.

Proof. Obviously the algebra $\mathcal{C}_{\omega}$ is $*$-invariant. To prove that it is a right coideal in Fun $_{q}(\mathcal{E}(2))$ is enough to perform straightforward calculations on the generators. We have:

$$
\begin{gathered}
\Delta v=v \otimes v \in \mathcal{E}_{\omega}(2) \otimes \mathcal{C}_{\omega} \\
\Delta m=1 \otimes m+\bar{v} \bar{n} \otimes \bar{v}-v n \otimes v \in \mathcal{E}_{\omega}(2) \otimes \mathcal{C}_{\omega} .
\end{gathered}
$$

To prove the linear independance of elements $v^{r} m^{s}$ let us first observe that $\mathcal{C}_{\omega}$ is a skew polynomial ring ([7]), and precisely $\mathcal{C}_{\omega}=R[m ; \delta]$ where $R=\mathbf{C}\left[v, v^{-1}\right]$ and $\delta(v)=\left(v^{2}-1\right)$. Thus it is possible to define the degree in $m$, that we will denote $d e g_{m}$, for every element in $\mathcal{C}_{\omega}$ and prove that if $n$ and $u$ are in $\mathcal{C}_{\omega}$ then:

$$
\operatorname{deg}_{m}(n u)=\operatorname{deg}_{m}(n)+\operatorname{deg}_{m}(u)
$$

It is then clear that there cannot be linear relations involving monomials with $\operatorname{deg}_{m} \neq 0$. On the other side linear relations involving only elements of $m$-degree 0 are linear relations in $\mathbf{C}\left[v, v^{-1}\right]$ and thus they should be trivial.

Let us observe that $\mathcal{C}_{\omega}$ being a skew polynomial ring is easily shown to verify good algebraic properties through very general arguments. For example it is a Noetherian integral domain.

In [6] it is shown how, in a fixed Hopf algebra, it is possible to construct a galoisian reciprocity between $*$-invariant subalgebras and right coideals on one side and Hopf $*$-ideals on the other side through the assignments:

$$
\begin{aligned}
& \Sigma: B \mapsto \mathcal{A}_{B}=\left\langle\left(S^{n}-\varepsilon \mathbf{1}\right)(b), b \in B, n \in \mathbf{Z}\right\rangle \\
& \Pi: \mathcal{A} \mapsto B_{\mathcal{A}}=\{b:(\pi \otimes i d) \circ \Delta(b)=1 \otimes b\}
\end{aligned}
$$


where $\pi$ is the projection from the whole Hopf algebra onto the quotient with respect to $\mathcal{A}$. It is then natural then to give the following:

(4.3) Definition. The closure of a quantum homogeneous space $\mathcal{C}$ in the Hopf algebra $\mathcal{H}_{q}$ is the homogeneous space $\mathcal{B}=\Pi \circ \Sigma(\mathcal{C})$.

(4.4) Proposition. The closure of the quantum cylinder $\mathcal{C}_{\omega}$ is the quantum homogeneous space corresponding to the quantum subgroup of non standard $\mathcal{E}_{\omega}(2)$ given by the Hopf-*-ideal $\mathcal{I}=\langle v-1, n-\bar{n}\rangle$.

Proof. The notation of last line of the proposition means that $\mathcal{I}$ is the ideal generated by elements enclosed in brackets. We want to verify that $\mathcal{I}$ is really a Hopf- $*$-ideal. First we have to verify that this ideal is a bilateral coideal i.e.:

$$
\begin{gathered}
\Delta \mathcal{I}=\mathcal{I} \otimes \mathcal{E}_{\omega}(2)+\mathcal{E}_{\omega}(2) \otimes \mathcal{I} \\
\varepsilon(\mathcal{I})=0
\end{gathered}
$$

It is enough to prove it for the generators. The second condition is trivial. The first follows from:

$$
\Delta(v-1)=v \otimes v-1 \otimes 1=(v-1) \otimes 1+v \otimes(v-1)
$$

$\Delta(n-\bar{n})=\bar{v} \otimes n+n \otimes 1-v \otimes \bar{n}-\bar{n} \otimes 1=\bar{v} \otimes(n-\bar{n})+(n-\bar{n}) \otimes 1+(\bar{v}-v) \otimes \bar{n}$

We next have to prove $S$-invariance. Again on generators we have:

$$
S(v-1)=(\bar{v}-1)=-\bar{v}(1-v) ; \quad S(n-\bar{n})=m=\bar{v}(\bar{n}-n)-(v-1)(\bar{v}+1) n
$$

so that $S(\mathcal{I}) \subset \mathcal{I}$. As for the $*$-structure we have:

$$
\begin{gathered}
(v-1)^{*}=\bar{v}-1=-\bar{v}(v-1) \in \mathcal{I} \\
(n-\bar{n})^{*}=n^{*}-\bar{n}^{*}=-(n-\bar{n}) \in \mathcal{I} .
\end{gathered}
$$

Next we have to verify that the closure of $\mathcal{C}_{\omega}$, as defined in (4.3), coincides with the set:

$$
B_{\mathcal{I}}=\left\{b \in \mathcal{E}_{\omega}(2):(\pi \otimes i d) \circ \Delta(b)=1 \otimes b\right\}
$$

where $\pi: \mathcal{E}_{\omega}(2) \rightarrow \mathcal{E}_{\omega}(2) / \mathcal{I}$ is the natural projection. Direct calculations show that indeed $v$ and $m$ belongs to this set and thus that $\mathcal{C}_{\omega}$ is contained in $B_{\mathcal{I}}$. For example:

$$
(\pi \otimes i d)(\Delta m)=1 \otimes m+\pi(\bar{v}(\bar{n}-n)) \otimes \bar{v}+\pi((\bar{v}-v) n) \otimes v
$$


The stabilizer ideal of $\mathcal{C}_{\omega}$ is:

$$
\mathcal{A}_{\mathcal{C}_{\omega}}=\Sigma\left(\mathcal{C}_{\omega}\right)=\left\langle S^{n}(c)-\varepsilon(c) 1, b \in \mathcal{C}_{\omega}, n \in \mathbf{Z}\right\rangle
$$

Let us note that $(S-\varepsilon \mathbf{1})(m)=n-\bar{n}$ and $(S-\varepsilon \mathbf{1})(\bar{v})=v-1$ so that the generators

of $\mathcal{I}$ belongs to $\mathcal{A}_{\mathcal{C}_{\omega}}$ proving $\mathcal{I} \subset \mathcal{A}_{\mathcal{C}_{\omega}}$. On the other hand from general arguments $\mathcal{C}_{\omega} \subset B_{\mathcal{I}}$ implies the opposite inclusion so that:

$$
\mathcal{A}_{\mathcal{C}_{\omega}}=\mathcal{I}
$$

This proves that the closure of $\mathcal{C}_{\omega}$ is exactly $B_{\mathcal{I}}$.

\section{Conclusions}

Analizing the Poisson structures and their Poisson homogeneous spaces for the euclidean group we have recovered the semiclassical limit of results in [2] about quantum homogeneous planes, showing that, in this limit there is a one-parameter family of planes, as it is the case for spheres ([12], [13]). Furthermore we have determined a quantization of a Poisson structure on the cylinder compatible with a quantum homogeneous structure with respect to nonstandard quantum euclidean group, showing that it can be recovered from an explicit quantum subgroup. Again we have showed a one parameter family of covariant Poisson structures on this space. The method of looking at the semiclassical limit situation to have hints towards the general quantum homogeneous spaces could perhaps give indication in all those situations in which the function algebra is explicitely given through generators and relations.

\section{Acknowledgements}

I am indebted with Dr. B. Drabant and Prof. R. Giachetti for useful discussions on the subject and to Prof. T.H. Koornwinder for careful reading of an early version of the manuscript.

\section{References.}

1. Ballesteros A., Celeghini E., Giachetti R., Sorace E. and Tarlini M., An Rmatrix approach to the quantization of the Euclidean group E(2). J. Phys. A: Math. Gen., 26, 7495 (1993). 
2. Bonechi F., Ciccoli N., Giachetti R., Sorace E., Tarlini M., Free q-Schrödinger equation from homogeneous spaces of the 2 -dim Euclidean quantum group. Comm. Math. Phys. in press.

3. Celeghini E., Giachetti R., Sorace E. and Tarlini M., 3-dim quantum groups from contractions of $S U_{q}(2)$. J. Math. Phys. 312548 (1990) and Contractions of Quantum Groups. Lecture notes in Mathematics 1510, 221 (Berlin, Springer, 1992).

4. Ciccoli N., Integer forms of nonsemisimple quantum groups, preprint, (1995).

5. Ciccoli N., Giachetti R., The euclidean quantum algebra at roots of unity. Lett. Math. Phys., in press.

6. Dijkhuizen M.S. and Koornwinder T.H., Quantum homogeneous spaces, duality and quantum 2-spheres. Geom. Ded. 52291 (1994).

7. Goodearl K. R., Letzter E. S., Mem. Amer. Math. Soc., 521, (1994).

8. Jordan D. A., Iterated skew polynomial rings and quantum groups. Journ. of Alg., 156, 194 (1993).

9. Koelink H. T., The quantum group of plane motion and the Hahn-Exton qBessel function. Duke Math. Journ., 76, 483 (1994).

10. Maslanka P., The $E_{q}(2)$ group via direct quantization of the Lie-Poisson structure and its Lie algebra. Journ. Math. Phys. 35, 1976 (1994).

11. Podles P., Quantum spheres. Lett. Math. Phys. 14, 193 (1987).

12. Sheu A. J. L., Quantization of the Poisson SU(2) and its Poisson homogeneous space, the 2-sphere. Comm. Math. Phys. 135, 217 (1991).

13. Sheu A. J. L., Quantum Poisson SU(2) and quantum Poisson spheres. Contemp. Math. 134, 247 (1992).

14. Smith. A. P., Quantum groups: an introduction and survey for ring theorists. in "Noncommutative rings", MSRI Publications, (Montgomery S. and Small L. W. eds.) 24, 131 (Berlin, Springer, 1992).

15. Vaksman L.L. and Korogodski L.I., An algebra of bounded functions on the quantum group of the motions of the plane and q-analogues of Bessel functions. Sov. Math. Dokl. 39, 173 (1989).

16. Woronowicz S., L., Unbounded elements affiliated with $C^{*}$ - algebras and non compact quantum groups. Comm. Math. Phys., 136, 399 (1991) and Quantum $S U(2)$ and $E(2)$ groups: contraction procedure . Comm. Math. Phys., 149, 637 (1992).

17. Zakrzewski S., Poisson homogeneous spaces. Proceedings of the Karpacz winter school on theoretical physics 1994, to appear. Also, hep-th 9412101. 